\title{
Hepatitis delta infection in Italian patients: towards the end of the story?
}

\author{
Tommaso Stroffolini ${ }^{1} \cdot$ Evangelista Sagnelli $^{2}$ - Caterina Sagnelli ${ }^{2} \cdot$ Maurizio Russello $^{3}$ Massimo De Luca ${ }^{4}$ \\ Floriano Rosina ${ }^{5}$ Bruno Cacopardo ${ }^{6}$ - Giuseppina Brancaccio ${ }^{7}$ - Caterina Furlan ${ }^{1}$ - Giovanni Battista Gaeta ${ }^{7}$ \\ Anna Licata $^{8} \cdot$ Piero Luigi Almasio $^{8} \cdot$ behalf of EPACRON study group
}

Received: 6 August 2016 / Accepted: 22 October 2016 / Published online: 5 November 2016

(C) Springer-Verlag Berlin Heidelberg 2016

\begin{abstract}
Background The endemicity of hepatitis delta virus infection in Italy has decreased in the last decades.

Aim To evaluate the current epidemiology of chronic delta infection in Italy and to compare the present findings with the corresponding figures from the previous studies.

Methods A cross-sectional study involving 16 referral centres scattered all over the country in 2014.

Results Out of the 513 hepatitis B surface antigen-positive subjects enrolled, 61 (11.9\%) were anti-delta positive, with
\end{abstract}

The members of EPACRON study group are listed in Acknowledgements.

Piero Luigi Almasio

piero.almasio@unipa.it

1 Department of Tropical and Infectious Diseases, Policlinico Umberto Primo, Rome, Italy

2 Department of Mental Health and Public Medicine, Second University of Naples, Caserta, Italy

3 Operative Unit of Hepatology and Gastroenterology, A.R.N.A.S. Garibaldi-Nesima of the Catania, Catania, Italy

4 Liver Unit, Division of Hepatology, Department of Transplantation, Cardarelli Hospital, Naples, Italy

5 Hepatogastroenterology Division, Ospedale Gradenigo, 10153 Turin, Italy

6 Department of Clinical and Experimental Medicine, Institute of Infectious Diseases, University of Catania, Catania, Italy

7 Infectious Diseases, Department of Mental and Physical Health and Preventive Medicine, Second University of Naples, Naples, Italy

8 Gastroenterology and Hepatology Unit, Biomedical Department of Internal and Specialized Medicine (Di. Bi.M.I.S.), University of Palermo, Piazza delle Cliniche, 2, 90127 Palermo, Italy a sex ratio $(\mathrm{M} / \mathrm{F})$ of 2.05 . The majority $(80.3 \%)$ of them was 50 years or older, while the proportion of subjects younger than 30 years of age was as low as $3.3 \%$. No difference was detected by geographical area of residence. The presence of liver cirrhosis was diagnosed in 52.4\% of cases. In comparison to previous studies, a further shift towards the oldest age groups and an increasing proportion of subjects having liver cirrhosis among all anti-delta-positive subjects are observed.

Conclusions Currently, hepatitis delta infection mostly affects old people who have an advanced but indolent liver disease, reflecting a survival effect. The defective hepatitis delta virus is near to disappear in the country, where it has been discovered in the second half of 70s.

Keywords HDV infection · HBsAg · Cirrhosis · Epidemiology

$\begin{array}{ll}\text { Abbreviations } \\ \text { HDV } & \text { Hepatitis delta virus } \\ \text { HBV } & \text { Hepatitis B virus } \\ \text { HBsAg } & \text { Hepatitis B surface antigen } \\ \text { anti-HDV } & \text { Antibodies to delta virus } \\ \text { HCC } & \text { Hepatocellular carcinoma } \\ \text { AFP } & \text { Alpha-fetoprotein }\end{array}$

\section{Introduction}

Hepatitis delta virus (HDV) discovered in the 1970s [1, 2] is a defective RNA virus requiring the helper function of hepatitis B virus (HBV) to establish its own infection. This discovery opened a new window for the knowledge of liver diseases adding important new issues even to the natural history of HBV infection. At that time, HDV was endemic 
in Italy, particularly in Southern areas, accounting for the majority of cases of juvenile cirrhosis [3]. High prevalence rates of HDV infection were worldwide reported, especially in Mediterranean areas, Eastern Europe, the Amazon basin and central Asia [4].

In Italy, after the introduction of the mass immunization campaign against HBV in 1991 for 3-month-old infants and for subjects of 12 years of age (the latter limited to the first 12 years of campaign), a control even of HDV infection was expected. In fact, since the second half of 80 s the incidence rate of acute HDV per million of inhabitants progressively declined from 3.1 cases in 1987 to 0.5 cases in $2004[5,6]$. The downward trend was mostly affecting the 15-24 years old age group, both reflected the decreased pool of chronic hepatitis B surface antigen (HBsAg) carriers [7], and the impact of mass immunization campaign against $\mathrm{HBV}$.

Similarly, the prevalence of antibodies to delta virus (anti-HDV) evaluated among cohorts of chronic HBsAg carriers referring Italian hospitals progressively declined over time, being $23.4 \%$ in 1987 [8], $14.0 \%$ in 1992 [9], $8.3 \%$ in 1997 [10], $5.7 \%$ in 2001 [11], and $8.1 \%$ in 2006 [12], respectively.

In this paper, we report the results of a further national survey performed in 2014 on the epidemiology of chronic HDV infection in Italy. Moreover, the present findings have been compared with the corresponding figures from the previous studies [8-12] to detect changes, if any.

\section{Materials and methods}

All HBsAg-positive subjects consecutively referring in 2014, as either inpatients or outpatients, to 16 Liver Units were recruited. Patients with acute B hepatitis were excluded from the study. The Liver Units participating were located throughout the country.

Liver cirrhosis was diagnosed by liver biopsy or on the presence of the characteristics of clinical, biochemical and ultrasound signs [13]. The diagnosis of hepatocellular carcinoma (HCC) was based on histological and/or imaging findings and on serum alpha-fetoprotein (AFP) levels, according to accepted criteria [14].

The collection of personal data was made in full compliance with the Italian law on personal data collection, and each patient gave his/her informed consent to participate. All procedures applied in the study were in accordance with the international guidelines, with the standards of human experimentation of the local Ethics Committees, and with the Helsinki Declaration of 1975, revised in 1983. Each patient signed informed consent for the collection of personal data, established in full agreement with the rules of the Ethic Committee of the coordinating centre (A.O.U.P. of Palermo, Italy). Patients who agreed to undergo liver biopsy signed informed consent before this procedure was performed. All patients were included only once, i.e. at the first observation during the study period. For each patient, demographic, clinical, and etiological data were recorded using e pre-coded questionnaire (Air-Tel telematica, Italy).

HBV serum markers and antibodies to hepatitis D virus (anti-HDV) were determined by commercial immunoenzymatic assays.

\section{Statistical analysis}

Categorical variables were summarized as absolute counts and proportions. Differences in proportion were evaluated by Chi square test or Fisher's exact test. A $p$ value $<0.05$ was considered to be significant. Crude odds ratios (ORs) for the association of anti-HDV positivity with different variables were calculated by univariate analysis. The reference category for ORs estimates was no exposure or the most favourable level of exposure.

\section{Results}

During the study period, 513 chronic HBsAg carriers were enrolled. All were tested for anti-HDV. Among them, 61 $(11.9 \%)$ had an anti-HDV-positive test result, a figure slightly higher than those reported in the studies performed since the year 1997 (Fig. 1). The main characteristics of the 61 anti-HDV-positive subjects are reported in Table 1. Sex ratio (male/female) was 2.05 , and the majority (80.3\%) of subjects were 50 years or older, while the proportion of subjects younger than 30 years of age was as low as 3.3\%. Twelve (19.7\%) of these 61 were anti-HCV positive. Liver biopsy was performed in $24.6 \%$ of cases, liver cirrhosis was diagnosed in 32 subjects $(52.4 \%)$ and in three of them $\mathrm{HCC}$ was detected.

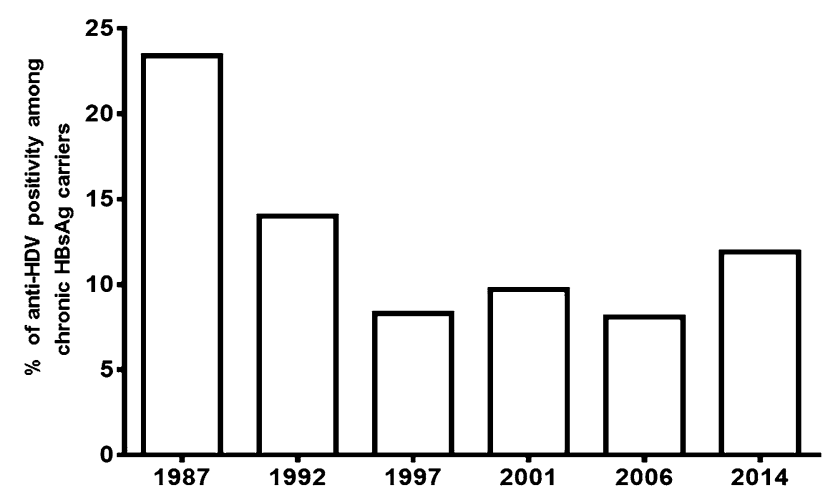

Fig. 1 Prevalence of anti-HDV positivity among chronic HBsAg carriers in Italy over time 
Table 1 Study population of 61 anti-HDV-positive subjects

\begin{tabular}{lr}
\hline Characteristic & \multicolumn{1}{c}{ No $(\%)$} \\
\hline Anti-HDV prevalence (HDV+/HBsAg+) & $61 / 513(11.9)$ \\
Sex ratio (M/F) & $41 / 20(2.05)$ \\
Age-distribution (years) & \\
$0-29$ & $2(3.3)$ \\
$20-49$ & $10(16.4)$ \\
$>49$ & $49(80.3)$ \\
Liver biopsy & $15(24.6)$ \\
Presence of cirrhosis & \\
\hline
\end{tabular}

a Three subjects with hepatocellular carcinoma

The age-distribution of anti-HDV-positive subjects in Italy over time plotted in Fig. 2 provides interesting information. During the period 1987-2014, the proportion of cases in the age group of 0-29 and 30-49 years strongly decreased from 39.8 to $3.3 \%$ and from 50.6 to $16.4 \%$, respectively. In contrast, the proportion of cases in subjects with 50 years or older increased from 9.6 to $80.3 \%$ (Table 2).

Anti-HDV prevalence was slightly higher in Northern/ Centre than Southern/Islands areas (13.6 vs. $12.0 \%$ ), a finding completely different from that observed in the previous surveys, which have shown a much higher prevalence than the latter regions.

Table 3 reports the sociodemographic characteristics of anti-HDV-positive subjects, the potential risk factors and the crude ORs with their 95\% Confidence Intervals (CI) derived from univariate analysis. The presence of cirrhosis resulted the sole variable significantly associated with HDV infection (OR 2.6, 95\% CI 1.5-4.5). Since only one variable resulted associated, multiple logistic regression
Table 2 Prevalence of anti-HDV positivity among chronic HBsAg carriers in Italy by geographical area, over time (1987-2014)

\begin{tabular}{llll}
\hline Year & \multicolumn{3}{l}{$\begin{array}{l}\text { Percent of patients with anti-HDV positivity among } \\
\text { chronic HBsAg carriers }\end{array}$} \\
\cline { 2 - 4 } & North/Centre (\%) & South/Islands (\%) & References \\
\hline 1987 & 13.1 & 26.6 & {$[7]$} \\
1992 & 9.0 & 18.5 & {$[8]$} \\
1997 & 1.9 & 11.3 & {$[9]$} \\
2001 & 6.6 & 8.3 & {$[10]$} \\
2006 & 8.7 & 6.7 & {$[11]$} \\
2014 & 13.6 & 12.0 & Present study \\
\hline
\end{tabular}

was not performed. Finally, the proportion of subjects with anti-HDV-positive patients with liver cirrhosis increased from $35.7 \%$ in 1987 to $52.4 \%$ in $2014(p<0.05)$ (Table 4$)$.

\section{Discussion}

The data herein reported complete the picture of chronic HDV infection in Italy over a very long period (19872014). Since several units participating in the current survey took also part in all previous investigations, current findings are suitable to be compared with the previous ones. Despite the current anti-HDV prevalence is slightly higher than that observed in the previous study performed in 2006 (11.9 vs. 8.1\%), we may assert that the spread of HDV infection in Italy is vanishing. Some lines of evidence support this evidence. Firstly, the age distribution of cases is mostly confined to people with more than 50 years of age, whilst people younger than 30 years account for only $3.3 \%$ of cases, Secondly, the proportion of
Fig. 2 Age distribution of antiHDV-positive subjects in HBs Ag carriers in Italy from 1987 to 2014

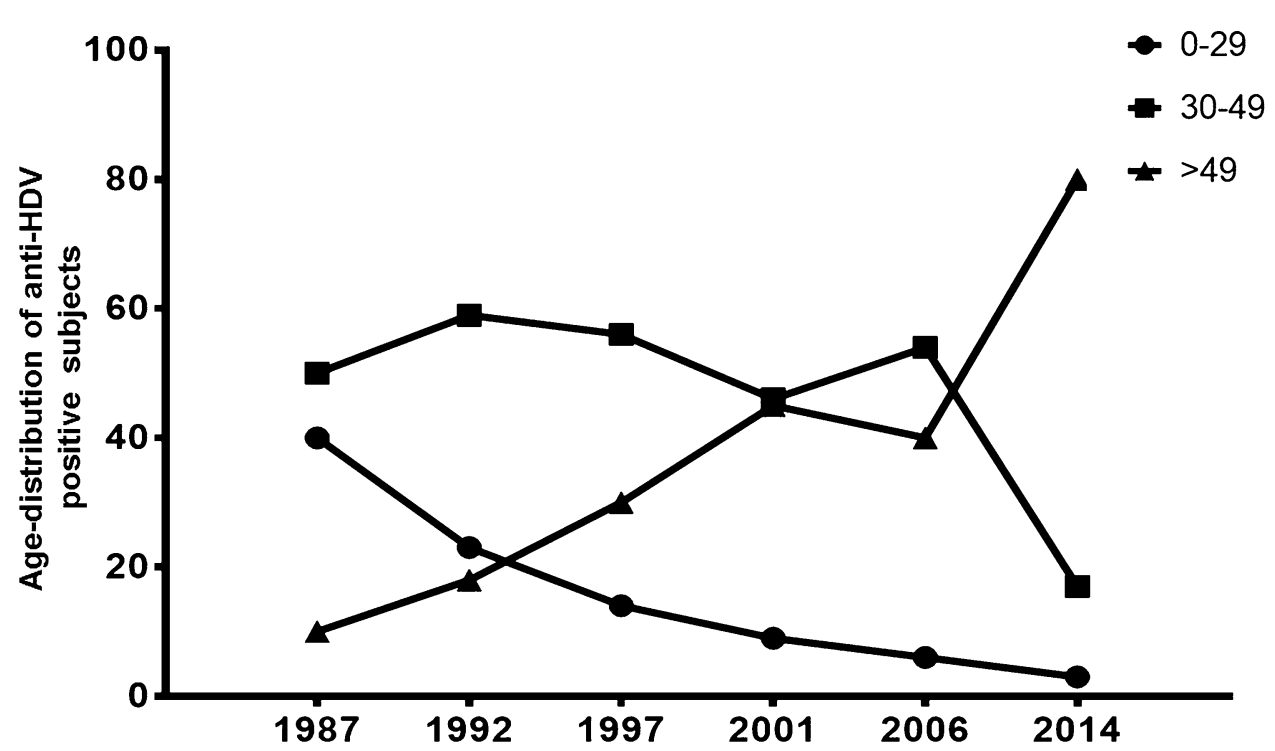


Table 3 Prevalence of anti-HDV positivity by sociodemographic characteristics and potential risk factors in $513 \mathrm{HBsAg}$-positive subjects, 2014

\begin{tabular}{|c|c|c|c|}
\hline Characteristic & HBsAg-positive No of cases & Anti-HDV-positive $\%$ of cases & OR $(95 \% \mathrm{CI})$ \\
\hline \multicolumn{4}{|l|}{ Sex } \\
\hline Females & 183 & 10.9 & 1 \\
\hline Males & 330 & 12.4 & $1.2(0.7-1.5)$ \\
\hline \multicolumn{4}{|l|}{ Age (years) } \\
\hline $0-29$ & 15 & 13.3 & 1 \\
\hline $30-49$ & 126 & 7.9 & $0.6(0.1-2.8)$ \\
\hline$>49$ & 371 & 13.2 & $0.99(0.2-4.5)$ \\
\hline \multicolumn{4}{|l|}{ Area of residence } \\
\hline North/Centre & 103 & 13.6 & $1.2(0.6-2.2)$ \\
\hline South/Islands & 375 & 12.0 & 1 \\
\hline \multicolumn{4}{|l|}{ Country of birth } \\
\hline Italy & 478 & 12.3 & $2.3(0.5-9.9)$ \\
\hline Outside Italy & 35 & 5.7 & 1 \\
\hline \multicolumn{4}{|l|}{ Educational level } \\
\hline High & 113 & 11.5 & 1 \\
\hline Medium-Low & 400 & 12.0 & $0.7(0.4-1.6)$ \\
\hline Blood transfusion & 51 & 11.8 & $0.99(0.4-2.4)$ \\
\hline IVDU & 32 & 9.4 & $1.3(0.4-4.5)$ \\
\hline Presence of cirrhosis & 166 & 19.3 & $2.6(1.5-4.5)$ \\
\hline
\end{tabular}

Crude odds ratio (OR) derived by univariate analysis

$I V D U$ intravenous drug use
Table 4 Proportion of subjects with liver cirrhosis among anti-HDV positive in Italy (1987-2014)

\begin{tabular}{lll}
\hline Year & Presence of cirrhosis No $(\%)$ & References \\
\hline 1987 & $130 / 364(35.7 \%)$ & {$[7]$} \\
1992 & $61 / 143(42.6 \%)$ & {$[8]$} \\
1997 & $30 / 69(43.5 \%)$ & {$[9]$} \\
2001 & $43 / 104(41.4 \%)$ & {$[10]$} \\
2006 & $37 / 95(38.9 \%)$ & {$[11]$} \\
2014 & $32 / 61(52.4 \%)$ & Present study \\
\hline
\end{tabular}

cirrhotic patients among all HDV-infected subjects has significantly increased over time reaching the current rate of $52.4 \%$, likely reflecting a so-called "survival effect". Taken together, these findings evidence a low current spread of HDV infection with an accumulation of cases having an advanced, slowly evolving disease. Actually, HDV infection in Italy is an ageing disease with a long-term survival, which explains the observed slightly higher overall antiHDV prevalence observed in the present study as compared to previous ones. This is in agreement with a follow-up study of subjects with biopsy-proven chronic HDV infection in two Italian medical centres showing a stable clinical cirrhosis for more than a decade [15].

The low proportion of cases in people younger than 30 years is an expected finding due to the mass vaccination campaign against HBV started in 1991, which depleted in the youngest age group the HBsAg carrier reservoir that represents the necessary substrate for the replication of HDV defective virus [16]. A second effect to explain the decline in $\mathrm{HBV}$ endemicity is the successful treatment of HBV infection with nucleoside analogues or other drugs with low adverse events since 1998. Indeed, this treatment has been extensively administered in Italy in the latter 15 years, but in the absence of a national register we may not be accurate on this point.

For several years, HDV infection has been characterized by a higher prevalence in Southern than in Northern Italian areas [8-11]. However, since the survey of 2006 [12], the prevalence has become higher in the latter ones. This evolving geographical pattern may reflect changes in the modes of transmission. Intrafamilial spread was the main source of HDV infection in Southern areas, whereas illicit drug abuse was the predominant risk factor in Northern Italy $[8,10,17]$. The reduced family size even in the South has affected the spread of infection in these areas, while i.v. drug use has continued to be practised, particularly in Northern areas, where it has been responsible for some epidemic peaks [6]. Although the majority of HDVinfected subjects have liver cirrhosis, its contribution to the entire pool of cirrhosis in Italy should be considered minimal. The last published survey [18] has shown that $13 \%$ of cirrhosis are HBV related and nearly $20 \%$ of these cirrhosis are caused by simultaneous HDV infection. Therefore, a little more than $2.5 \%$ of all cirrhosis cases in Italy are attributable to HDV infection. 
The decreasing endemicity of HDV infection in Italy has led to a downgrading alert about the risk of its spreading. In fact, it has been shown that $35.3 \%$ of HBsAg-positive subjects in Southern Italy in 2005 [19], and as many as $53.8 \%$ of acute HBV cases during the period 1993-2004 [6] were not tested for anti-HDV. More attention should be addressed to HDV infection testing for all subjects who are found to be HBsAg positive.

In conclusion, current HDV infection in Italy mostly affects old people who have an advanced but indolent liver disease, as a consequence of survival effect. Based on this epidemiological survey, we can hypothesize that this defective virus is near to disappear in the Country where it has been firstly discovered in the second half of 70 s.

Acknowledgements The members of EPACRON study group: Coordinating group: Piero Luigi Almasio, Giovanni Battista Gaeta, Evangelista Sagnelli, Tommaso Stroffolini. Peripheral centres: Angelo Andriulli (Gastroenterology Unit, Fondazione "Casa Sollievo della Sofferenza" IRCCS Hospital, San Giovanni Rotondo, Foggia, Italy), Sergio Babudieri (Clinical of Infectious Disease, University of Sassari, 07100 Sassari, Italy), Giuseppina Brancaccio, Bruno Cacopardo, Guido Colloredo (Department of Internal Medicine, San Pietro Hospital, Ponte San Pietro, Italy), Nicola Coppola (Department of Mental Health and Public Medicine, Second University of Naples, Italy), Massimo De Luca, Caterina Furlan, Anna La Licata (Gastroenterology and Hepatology Unit, Di.Bi.MI.S. University of Palermo, Italy), Filomena Morisco (Department of Clinical Medicine and Surgery, Gastroenterology Unit, University of Naples "Federico II", Naples, Italy), Mario Pirisi (Department of Translational Medicine, Università del Piemonte Orientale, Novara, Italy) Mariantonietta Pisaturo (Division of Infectious Diseases, AORN Sant'Anna e San Sebastiano di Caserta, 81100, Caserta, Italy), Floriano Rosina, Maurizio Russello, Caterina Sagnelli, Teresa Santantonio (Department of Clinical and Experimental Medicine, University of Foggia, 71100 Foggia, Italy), Antonina Smedile (Department of Gastroenterology, Molinette Hospital, C.so Bramante 88, 10126 Turin, Italy).

\section{Compliance with ethical standards}

Conflict of interest All the authors of the manuscript declare that they have no conflict of interest in connection with this paper.

Financial support An unrestricted grant for the study was provided by Gilead.

\section{References}

1. Rizzetto M, Canese MG, Aricò S, Crivelli O, Trepo C, Bonino $\mathrm{F}$, et al. Immunofluorescence detection of new antigen-antibody system (delta/anti-delta) associated to hepatitis B virus in liver and in serum of HBsAg carriers. Gut. 1977;18:997-1003.

2. Sureau C, Negro F. The hepatitis delta virus: replication and pathogenesis. J Hepatol. 2016;64:S101-16.

3. Smedile A, Lavarini C, Farci P, Aricò S, Marinucci G, Dentico $\mathrm{P}$, et al. Epidemiologic patterns of infection with the hepatitis B virus-associated delta agent in Italy. Am J Epidemiol. 1983;117:223-9.

4. Rizzetto M, Purcell RH, Gerin JL. Epidemiology of HBVassociated delta agent: geographical distribution of anti-delta and prevalence in polytransfused HBsAg carriers. Lancet. 1980;1(8180):1215-8.

5. Stroffolini T, Ferrigno L, Cialdea L, Catapano R, Palumbo F, Novaco F, et al. Incidence and risk factors of acute Delta hepatitis in Italy: results from a national surveillance system. SEIEVA Collaborating Group. J Hepatol. 1994;21:1123-6.

6. Mele A, Mariano A, Tosti ME, Stroffolini T, Pizzuti R, Gallo G, et al. Acute hepatitis delta virus infection in Italy: incidence and risk factors after the introduction of the universal anti-hepatitis B vaccination campaign. Clin Infect Dis. 2007;44:e17-24.

7. Stroffolini $\mathrm{T}$. The changing pattern of hepatitis B virus infection over the past three decades in Italy. Dig Liver Dis. 2005;37:622-7.

8. Sagnelli E, Stroffolini T, Ascione A, Bonino F, Chiaramonte M, Colombo M, et al. The epidemiology of hepatitis delta infection in Italy. Promoting Group. J Hepatol. 1992;15:211-5.

9. Sagnelli E, Stroffolini T, Ascione A, Chiaramonte M, Craxì A, Giusti G, et al. Decrease in HDV endemicity in Italy. J Hepatol. 1997;26:20-4.

10. Gaeta GB, Stroffolini T, Chiaramonte M, Ascione T, Stornaiuolo G, Lobello S, et al. Chronic hepatitis D: a vanishing disease? An Italian multicenter study. Hepatology. 2000;32:824-7.

11. Sagnelli E, Stroffolini T, Mele A, Imparato M. Almasio PL; Italian Hospitals' Collaborating Group. Chronic hepatitis B in Italy: new features of an old disease - approaching the universal prevalence of hepatitis B e antigen-negative cases and the eradication of hepatitis D infection. Clin Infect Dis. 2008;46:110-3.

12. Gaeta GB, Stroffolini T, Smedile A, Niro G, Mele A. Hepatitis delta in Europe: vanishing or refreshing? Hepatology. 2007:46:1312-3.

13. Rosina F, Conoscitore P, Cuppone R, Rocca G, Giuliani A, Cozzolongo $\mathrm{R}$, et al. Changing pattern of chronic hepatitis $\mathrm{D}$ in Southern Europe. Gastroenterology. 1999;117:161-6.

14. Bonino F, Caporaso N, Dentico P, Marinucci G, Valeri L, Craxì $\mathrm{A}$, et al. Familiar clustering and spreading of hepatitis delta virus infection. J Hepatol. 1985;1:221-6.

15. Stroffolini T, Sagnelli E, Almasio $\mathrm{P}$, Ferrigno L, Craxì A, Mele A, et al. Characteristics of liver cirrhosis in Italy: results from a multicenter national study. Dig Liver Dis. 2004;36:56-60.

16. Stroffolini T, Guadagnino V, Rapicetta M, Menniti Ippolito F, Caroleo B, De Sarro G, et al. The impact of a vaccination campaign against hepatitis $B$ on the further decrease of hepatitis $B$ virus infection in a southern Italian town over 14 years. Eur J Intern Med. 2012;23:e190-2.

17. Raimondo G, Isgrò G, Caccamo G, Pollicino T, Squadrito G, Calabrian HBV. Study Group. Is there a downgrading in the alert about the hepatitis B virus infection in Italy? Dig Liver Dis. 2007:39:257-61.

18. Gaiani S, Gramantieri L, Venturoli N, Piscaglia F, Siringo S, D'Errico A, et al. What is the criterion for differentiating chronic hepatitis from compensated cirrhosis? A prospective study comparing ultrasonography and percutaneous liver biopsy. J Hepatol. 1997;27:979-85.

19. Bruix J, Sherman M, Llovet JM, Beaugrand M, Lencioni R, Burroughs AK, et al. Clinical management of hepatocellular carcinoma. Conclusions of the Barcelona-2000 EASL conference. European Association for the Study of the Liver. J Hepatol. 2001;35:421-30. 